\title{
Fundus Autofluorescence in Age-related Macular Degeneration
}

\author{
Angelica Ly*, Lisa Nivison-Smith ${ }^{\dagger}$, Nagi Assaad ${ }^{\ddagger}$, and Michael Kalloniatis ${ }^{\S}$
}

\begin{abstract}
Fundus autofluorescence (FAF) provides detailed insight into the health of the retinal pigment epithelium (RPE). This is highly valuable in age-related macular degeneration (AMD) as RPE damage is a hallmark of the disease. The purpose of this paper is to critically appraise current clinical descriptions regarding the appearance of AMD using FAF and to integrate these findings into a chair-side reference. A wide variety of FAF patterns have been described in AMD, which is consistent with the clinical heterogeneity of the disease. In particular, FAF imaging in early to intermediate AMD has the capacity to reveal RPE alterations in areas that appear normal on funduscopy, which aids in the stratification of cases and may have visually significant prognostic implications. It can assist in differential diagnoses and also represents a reliable, sensitive method for distinguishing reticular pseudodrusen. FAF is especially valuable in the detection, evaluation, and monitoring of geographic atrophy and has been used as an endpoint in clinical trials. In neovascular AMD, FAF reveals distinct patterns of classic choroidal neovascularization noninvasively and may be especially useful for determining which eyes are likely to benefit from therapeutic intervention. FAF represents a rapid, effective, noninvasive imaging method that has been underutilized, and incorporation into the routine assessment of AMD cases should be considered. However, the practicing clinician should also be aware of the limitations of the modality, such as in the detection of foveal involvement and in the distinction of phenotypes (hypo-autofluorescent drusen from small areas of geographic atrophy).
\end{abstract}

(Optom Vis Sci 2017;94:246-259)

Key Words: age-related macular degeneration, autofluorescence, chair-side reference, drusen, imaging

A ge-related macular degeneration (AMD) is a degenerative ocular condition and a leading cause of blindness and visual impairment worldwide with an estimated global prevalence of $8.69 \% .^{1}$ The condition is phenotypically heterogeneous, and experience has highlighted difficulties in distinguishing clinical signs typical of AMD (such as drusen) from other etiologies

*BOptom(Hons), GradCertOcTher

${ }^{\dagger} \mathrm{BSc}$ (Hons), $\mathrm{PhD}$

${ }^{*}$ MBBS, MBiomedE, FRANZCO

${ }^{\S} \mathrm{MScOptom}, \mathrm{PhD}, \mathrm{FAAO}$

Centre for Eye Health, Sydney, New South Wales(AL, LN-S, NA, MK); School of Optometry and Vision Science, University of New South Wales Australia, Sydney, New South Wales(AL, LN-S, MK); and Department of Ophthalmology, Prince of Wales Hospital, Randwick, New South Wales, Australia(NA).

This is an open-access article distributed under the terms of the Creative Commons Attribution-Non Commercial-No Derivatives License 4.0 (CCBYNC-ND), where it is permissible to download and share the work provided it is properly cited. The work cannot be changed in any way or used commercially without permission from the journal. or normal changes of the retina. ${ }^{2}$ This is problematic as the accurate detection and classification of AMD cases is fundamental to optimal patient care, and failure to do this represents a burden to the patient and to the health care system.

The last two decades have seen the advent of multiple imaging technologies, which allow for a variety of approaches to investigating macular health and disease. These new imaging modalities have been rapidly adopted by eye-care professionals in general practice and may be more precise than traditional methods at detecting morphological changes in early disease. One such modality, fundus autofluorescence imaging, can noninvasively provide detailed insights into the health of the retinal pigment epithelium (RPE). ${ }^{3}$ This is highly valuable in AMD as RPE damage is a hallmark of the disease.

The heterogeneity of AMD also means that the detection rate of early disease and the accuracy of diagnosis and staging using advanced imaging are likely to depend on the experience and knowledge of the examiner. ${ }^{4}$ Consequently, the purpose of this article is to collate and synthesize current descriptions relating to the appearance of AMD using fundus autofluorescence imaging. 
A systematic literature review was conducted, the information applied to clinical cases, and findings integrated into a chair-side reference chart.

\section{METHODS}

\section{Literature Review}

A systematic literature review was performed by searching the National Institutes of Health's PubMed database using the following combination of keywords and their relevant truncations and abbreviations: age-related macular degeneration, age-related maculopathy, and autofluorescence. Ten articles were identified through the bibliographies of clinical guidelines on $\mathrm{AMD}^{5-8}$ available in the public domain. Exclusion criteria were predetermined using a specific subject focus: qualitative evaluation of normal and abnormal fundus autofluorescence findings in vivo in AMD and related conditions. Derivatives of the techniques (such as quantitative fundus autofluorescence and near-infrared fundus autofluorescence) were excluded. Data extraction was facilitated by coding using NVivo qualitative data analysis software (QSR International Pty Ltd. version 10, 2014).

Sixty-four articles published between 2005 and 2014 were included in the final review. Non-English articles were not specifically excluded. However, a translated full text was not always accessible so the abstract only was considered. The appraised articles were predominantly retrospective, cross-sectional, observational studies. Fourteen studies $(22 \%)^{9-22}$ were prospectively designed, five $(8 \%)^{9,13,17,23,24}$ were longitudinal or natural history studies, whereas two articles $(3 \%)^{20,25}$ enrolled a control group.

\section{Technical Principles of Fundus Autofluorescence Imaging}

The fundus autofluorescence signal derives predominantly from lipofuscin granules in the RPE. The fluorophore that specifically causes fundus autofluorescence found in lipofuscin is $N$ retinylidene- $N$-retinylethanolamine (A2E), which accumulates as a by-product of incomplete degradation of photoreceptor outer segments in the RPE. ${ }^{3}$ Excessive accumulation of lipofuscin is recognized as a common pathogenetic pathway in various retinal diseases (including AMD) that precedes photoreceptor degeneration. Lipofuscin granules may also accumulate with age. ${ }^{26} \mathrm{Al}-$ ternative locations for fundus autofluorescence such as in the outer retina, ${ }^{27,28}$ photoreceptor outer segment, ${ }^{10}$ and subretinal space ${ }^{28}$ and sources of fundus autofluorescence such as macrophages in the new vessel complex, ${ }^{10}$ choroidal components, ${ }^{27}$ and the sclera have been suggested, but the exact nature and relative contributions of fundus fluorophores have not been fully characterized. ${ }^{4,12}$ Consequently, fundus autofluorescence imaging provides information different to that gained from other modalities (such as color fundus photography or fluorescein angiography) $9,22,23,27$ and has previously been shown to be underutilized. ${ }^{27,29}$

Most commonly, fundus autofluorescence images can be obtained clinically using a confocal scanning laser ophthalmoscope and blue light excitation (wavelength of $488 \mathrm{~nm}$, delivered using an argon blue or optically pumped solid-state laser). Emission is recorded in vivo using a barrier filter between 500 and $700 \mathrm{~nm}$ with a detection efficiency of $85 \%$ (Spectralis Heidelberg
Retina Angiograph HRA classic or HRA 2; Heidelberg Engineering, Heidelberg, Germany). ${ }^{3}$ Alternatively, clinical fundus autofluorescence images may be obtained relatively inexpensive$1 \mathrm{y}^{30}$ using a fundus camera and standardized filters described by Spaide ${ }^{31}$ providing excitation in the green spectrum (500-610 $\mathrm{nm})$. In that case, emission is recorded in the yellow-orange spectrum (675-715 nm). Optomap ultra-widefield imaging (Optos, Dunfermline, Scotland, UK) is a third example of fundus autofluorescence imaging that uses a green excitatory beam of $532 \mathrm{~nm}$ and emission wavelengths between 540 and $800 \mathrm{~nm} .^{32}$

Forty-nine of sixty-four (75\%) articles included in this review used the HRA classic or HRA2 as the primary method for the acquisition of images; 10/64 (16\%) applied a modified fundus camera including one article ${ }^{30}$ that utilized both methods. Two studies $(3 \%)^{20,33}$ used Optomap whereas one article ${ }^{34}$ used the F-10 confocal scanning laser ophthalmoscope (Nidek Co., Gamagory, Japan). Three articles failed to specify the instrument used. Consequently, blue light autofluorescence (compared to green light) using confocal scanning laser ophthalmoscopy represents the more well-established method of fundus autofluorescence imaging and has been used in quintessential classification schemes. 22,27,29

The final output of fundus autofluorescence imaging appears as an en face image of naturally or pathologically occurring fluorophores, which allows the user to visualize the spatial distribution and intensity of autofluorescence across the fundus. Images may be acquired efficiently in a clinical setting, though typically necessitates pupillary dilation (with the exception of Optomap). The field of view captured in fundus autofluorescence images may vary between 30 and 200 degrees depending on the specific instrument employed. At present, clinical evaluation of case images is primarily performed qualitatively and may require post-acquisition adjustments in brightness and contrast. ${ }^{15}$

\section{Case Images}

A retrospective review of noteworthy cases that presented between 2010 and 2015 to the Centre for Eye Health (CFEH) Sydney, Australia was also performed to identify representative cases. All patient records and images were independently reviewed by at least two experienced clinicians in concordance with CFEH's diagnostic protocols. Illustrative cases were chosen based on the consistency of the fundus autofluorescence image to previous descriptions in the literature. Fundus autofluorescence images were acquired as part of a standardized testing protocol using the Spectralis HRA $2(5 \mu \mathrm{m} /$ pixel lateral digital resolution, $768 \times 768$ pixels minimum image size, $30^{\circ}$ field of view) or Optomap 200Tx $\left(14 \mu \mathrm{m} /\right.$ pixel resolution, $3000 \times 3000$ pixels image size, $200^{\circ}$ field of view). HRA2 images were acquired in high-resolution mode, centered on the macula, and the automatic real time or mean image feature was also applied on multiple frames to generate a single image of amplified signal and reduced noise. ${ }^{26,35}$

Final images were all reviewed by an ophthalmologist with a medical retina subspecialty (NA). Staging of AMD was determined using the Beckman Initiative for Macular Research Classification scheme. ${ }^{36}$ Finally, a chair-side reference chart was devised. Patient written consent was obtained in accordance with the Declaration of Helsinki and approved by the Biomedical Human Research Ethics Advisory Panel of the University of New South Wales, Australia. 


\section{RESULTS}

\section{Fundus Autofluorescence Imaging in Normal Eyes}

The normal fundus exhibits diffuse, homogenous autofluorescence with large retinal blood vessels and the optic disc appearing as shadows of lower signal (Fig. 1A-D). ${ }^{12,22,27,37}$ The central macula typically shows a gradual reduction in fundus autofluorescence approaching the fovea, ${ }^{27,30}$ which relates to the absorption of short wavelength light by macular pigment (primarily lutein and zeaxanthin located in the neurosensory retina). ${ }^{25,27,35,38,39} \mathrm{~A}$ similar decline in fundus autofluorescence occurs toward the mid-peripheral fundus. ${ }^{12}$ Choroidal vessels should only be visible in the complete absence of the RPE (geographic atrophy ${ }^{12}$ ) or with use of a longer excitation wavelength (green light, owing to greater depth of penetration). ${ }^{30}$

Wide-field fundus autofluorescence imaging carries the potential to detect more widespread disease than narrower fields, ${ }^{27}$ and the peripheral fundus should appear as a homogeneous background. ${ }^{20,33}$
Although eyes with neovascular AMD have a significantly higher frequency of peripheral abnormalities compared with non-neovascular AMD or normal eyes, ${ }^{20,33}$ the prognostic implications of peripheral abnormalities visualized using ultra-widefield fundus autofluorescence imaging in AMD are unknown. ${ }^{20,33}$

\section{Fundus Autofluorescence Imaging of Early to Intermediate AMD}

The Beckman initiative for macular research classification committee ${ }^{36}$ described three stages of AMD: early, intermediate, and late (advanced). Using that scheme, cases presenting with small drusen (drupelets, $<63 \mu \mathrm{m}$ in size) only are classified as having normal aging changes. Medium drusen $(\geq 63$ to $<125 \mu \mathrm{m})$ are classified as early AMD, whereas large drusen $(\geq 125 \mu \mathrm{m})$ or pigmentary abnormalities associated with at least medium drusen
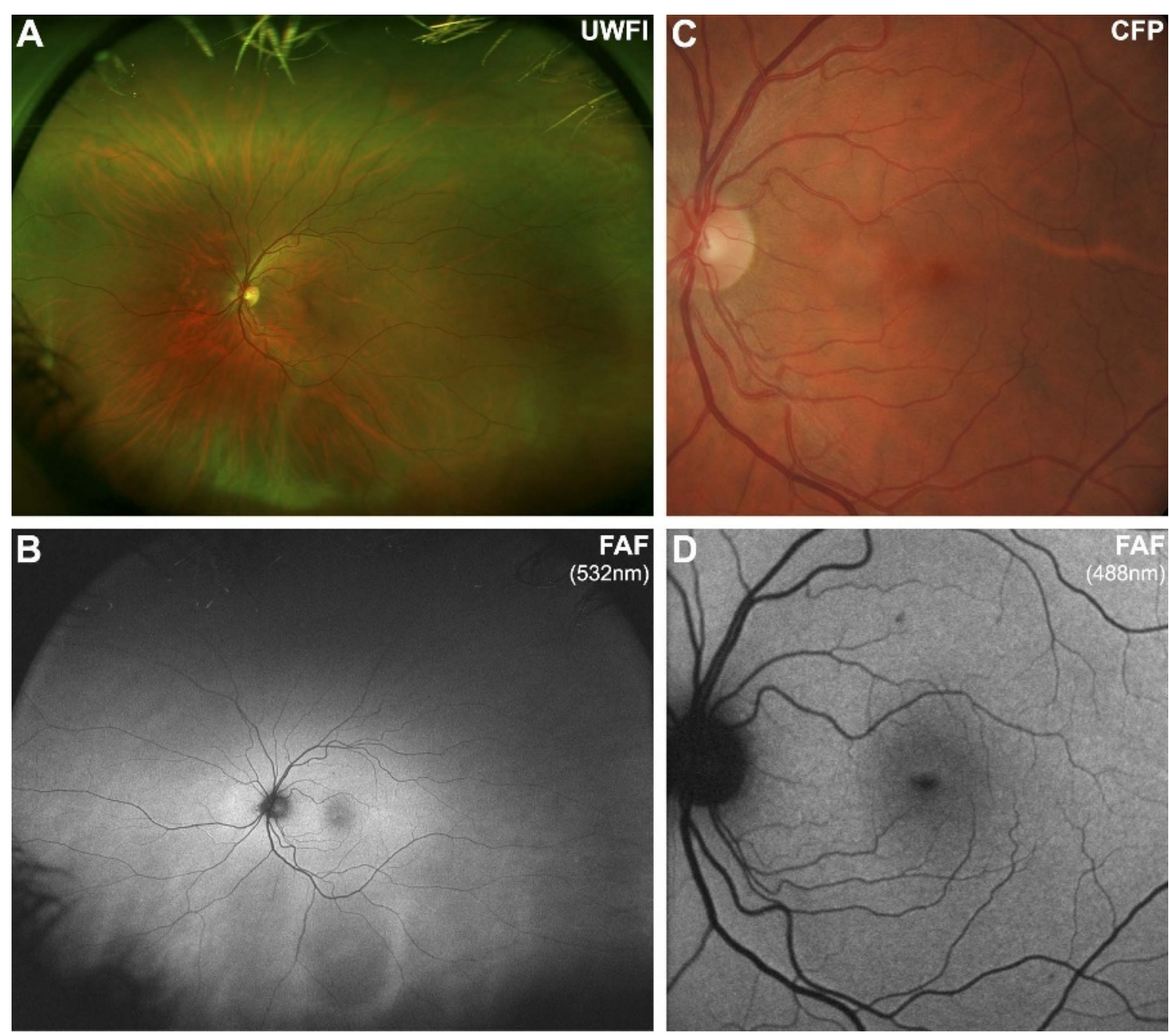

\section{FIGURE 1.}

Imaging results from a 43-year-old Caucasian female with a normal macula. Note that the normal fundus displays diffuse, homogenous autofluorescence with large retinal blood vessels and the optic disc appearing as shadows of hypo-autofluorescence. The central macula shows a gradual reduction in autofluorescence approaching the fovea, due to macular pigment. A, Optomap composite ultra-widefield image representing approximately 200 degrees of the fundus. B, Corresponding ultra-widefield fundus autofluorescence (FAF) image showing the potential of this device to detect more widespread disease than narrower fields. C, Retinal photograph cropped to size. D, FAF image obtained using the Heidelberg Retinal Angiograph 2 with a 30-degree field of view, which was the primary method of image acquisition in 49/64 articles included in this review. UWFI, ultra-widefield image; FAF, fundus autofluorescence; CFP, color fundus photograph. 
indicate intermediate AMD. These signs may also occur in late stage disease, and their identification is important for assessing the individual's risk of progression to late AMD. The following subsections will describe the optical basis of fundus autofluorescence changes in signs of early to intermediate AMD: drusen, pigmentary changes, and reticular pseudodrusen.

\section{Drusen}

Drusen exhibit normal, hyper-, or hypo-autofluorescent patterns on fundus autofluorescence imaging. ${ }^{27,28}$ This likely reflects the variable composition and size of drusen (and related fluorophores), and to their non-uniform effect on overlying RPE and photoreceptors. ${ }^{27,28}$ Consequently, the fundus autofluorescence status of drusen may reflect the wellbeing of overlying photoreceptors. ${ }^{28}$ Small and medium-sized drusen are especially likely to display a variable fundus autofluorescence effect, ${ }^{4,13,25,27,40}$ and may often present unnoticed (Fig. 2A-D, $2 \mathrm{G}, \mathrm{H}) .{ }^{28}$ Such changes likely relate to difficulties in imaging smaller lesions or changes in the drusen as they enlarge. ${ }^{4}$

Large (especially soft) drusen typically display pronounced, relatively well-defined hyper-autofluorescence (Fig. 2E, F). ${ }^{4,13,25,27,39,40}$
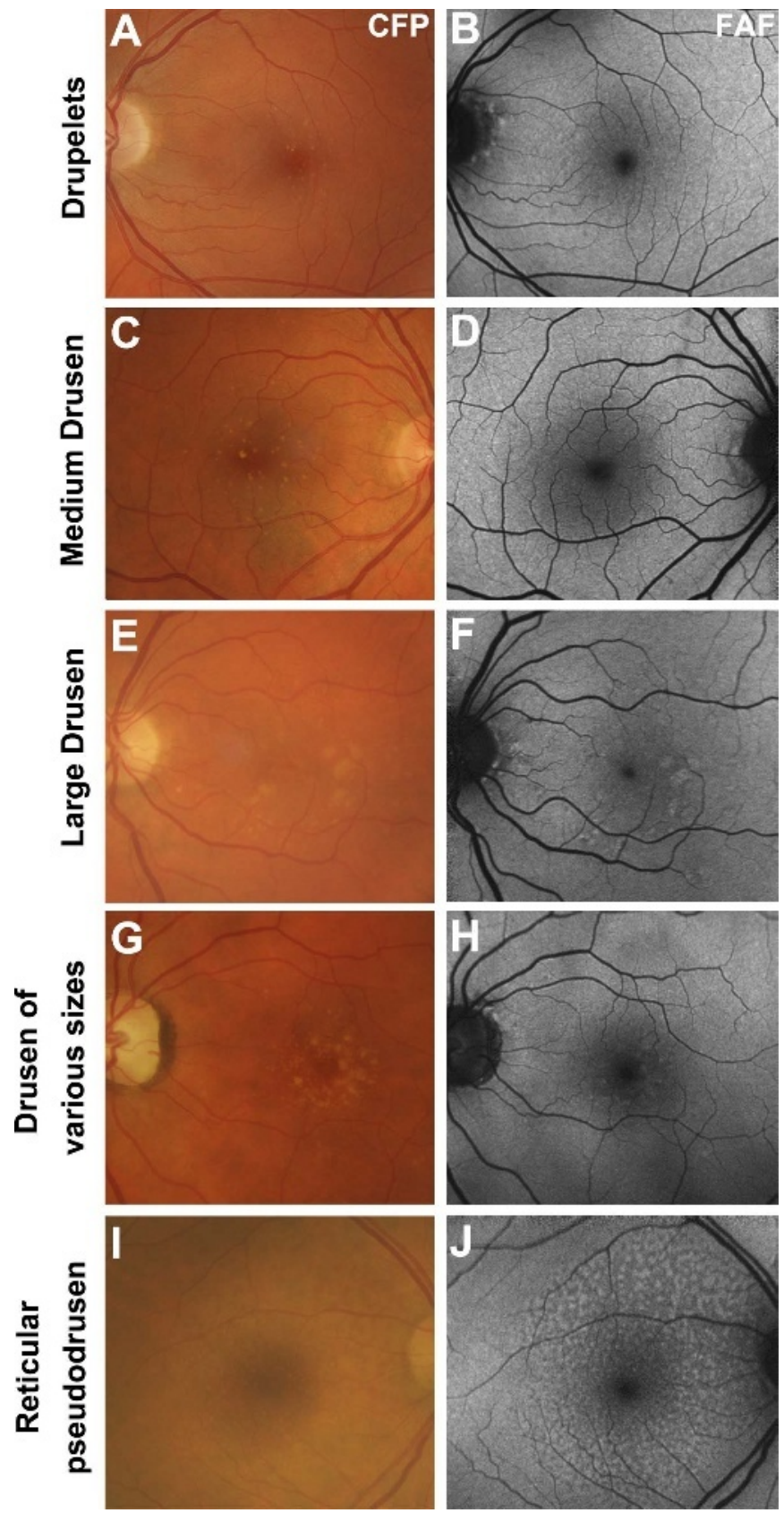

\section{FIGURE 2.}

The appearance of drusen subtypes using fundus autofluorescence. All images are presented in pairs. A-B, Drupelets (small drusen) and (C-D) mediumsized drusen causing a negligible effect on the fundus autofluorescence image. E-F, Large, soft indistinct drusen, which appear as well-defined patches of hyper-autofluorescence. G-H, Drusen varying in size from small to large, showing their variable effects. I-J, Reticular pseudodrusen, appearing as multiple, clustered, regularly networked, round areas of low-contrast hypo-autofluorescence. Abbreviations as in Fig. 1. 
Drusenoid pigment epithelial detachment displays a patchy pattern of hyper- and hypo-autofluorescence. ${ }^{39}$ These alterations relate to the presence of fluorescent material within the drusen itself or to the accumulation of lipofuscin in RPE overlying the drusen. ${ }^{4,25}$ Conversely, spots of reduced fundus autofluorescence may represent regressed drusen ${ }^{21}$ or reduced phagocytosis (and lipofuscin) in degenerating RPE, preceding geographic atrophy. ${ }^{12}$

\section{Pigmentary Changes}

Hyperpigmentary changes in AMD typically appear as focal, linear (Fig. 3B), and/or lace-like increases in fundus autofluorescence intensity $4,13,27,29$ and never display reticular, focal plaque-like, or normal fundus autofluorescence patterns. ${ }^{40}$ The alterations have been attributed to melanolipofuscin. ${ }^{4,40}$ However, the deposition of melanolipofuscin may be non-uniform and consequently, hyperpigmentary changes in AMD that demonstrate relatively low levels of increased fundus autofluorescence likely comprise melanin only. ${ }^{4}$

Hypopigmentary changes in AMD commonly correlate with decreased fundus autofluorescence due to an absence or degeneration of RPE and associated reductions in lipofuscin content. ${ }^{13,27}$ Conversely, hyper-autofluorescence in the presence of hypopigmentation on fundus photography may relate to an unmasking of normal lipofuscin levels in hypopigmented areas. ${ }^{4}$

\section{Reticular Pseudodrusen}

Fundus autofluorescence imaging has shown a high sensitivity (at least $86.5 \%^{24,41}$ ) for the detection of reticular pseudodrusen, also termed reticular fundus autofluorescence patterns. Fundus autofluorescence imaging of AMD patients should be carefully inspected for reticular pseudodrusen (especially in the superior outer macula and superotemporal to the macula ${ }^{16,27,42-44}$ ) as the reticular pseudodrusen phenotype in early or intermediate AMD significantly increases the risk of progression. ${ }^{24,33,43,44}$ Accordingly, their presence should alert the eye-care professional to conduct more regular follow-up. ${ }^{45}$ Reticular fundus autofluorescence patterns typically co-localize with a reticular pattern of small, yellowwhite, round, or oval lesions using funduscopy and/or color fundus photography (most apparent in red-free or blue light). ${ }^{14,43,46}$

Reticular pseudodrusen appear as multiple, clustered, small (between 50 and $400 \mu \mathrm{m}$ diameter, often $<200 \mu \mathrm{m}$ ), regularly networked, homogeneous, round, or oval areas of low-contrast hypo-autofluorescence surrounded by normal fundus autofluorescence (Fig. 2I, J). ${ }^{4,13,16,24,27,43}$ Although the exact reasons for reticular pseudodrusen hypo-autofluorescence are unknown, it may relate to a subretinal location of the deposits that blocks reflectivity of lipofuscin in the RPE. ${ }^{41,43}$ Reticular pseudodrusen never appear to involve the central zone using fundus autofluorescence imaging ${ }^{42}$ due to blocking of the excitation light by macular pigment. ${ }^{41,42}$

Careful evaluation of individual lesions may show a target appearance (iso-autofluorescent core and surrounding hypoautofluorescent halo). ${ }^{14}$ The iso-autofluorescent core of reticular pseudodrusen suggests central lipofuscin deposition or may relate to increased signal or transmission through the subretinal material indicating variations in drusen type/formation. ${ }^{14}$ Alternatively, visualization of the core may only be present if the confocal scanning laser ophthalmoscope image is focused on the centre of the drusen base. ${ }^{14}$ An uncommon, hyper-autofluorescent variant type of subretinal drusenoid deposits with similar characteristics to reticular pseudodrusen has also been described and is speculated to represent small foci of vitelliform material or a transient form of reticular pseudodrusen. ${ }^{46}$

Fundus autofluorescence imaging of reticular pseudodrusen using confocal scanning laser ophthalmoscopy has shown a high sensitivity and exceptional inter- and intra-observer agreement. ${ }^{16,24,41,45}$ This modality also displays specificity scores between $92 \%{ }^{24}$ and $95 \% .{ }^{41}$ However, subtle reticular fundus autofluorescence patterns may be difficult to differentiate from other AMD phenotypes when using this imaging modality alone. ${ }^{41,44,45}$ Consequently, confirmation of the phenotype may be best determined using an instrument with higher specificity, such as optical coherence tomography (OCT) or red-free images. ${ }^{4,24}$

\section{Stratification of Early to Intermediate AMD Phenotypes Using Fundus Autofluorescence}

In early AMD, fundus autofluorescence imaging has the capacity to reveal alterations in areas that appear normal on funduscopy. ${ }^{13,27,37,40,47}$ In eyes with intermediate AMD, the predominant finding is spots (punctate changes) of increased fundus autofluorescence (87.9\%). ${ }^{12}$ Spots of reduced fundus autofluorescence (26.7\%) and lines of increased fundus autofluorescence $(19.8 \%)$ may also be observed. ${ }^{12}$

The International Fundus Autofluorescence Classification Group (IFAG) ${ }^{27}$ described a classification scheme for a range of distinct fundus autofluorescence patterns associated with early and intermediate AMD including: normal, minimal change, focal increased, patchy, linear, lace-like, reticular, or speckled. Their scheme showed relatively high intra- and inter-observer agreement and prognostic implications were suggested. Other descriptions that have been reported include focal confluent, ${ }^{37}$ focal plaque-like, ${ }^{13}$ and scattered. ${ }^{37}$ The prevalence of each phenotype seems to vary between studies ${ }^{17,28,40}$ and may depend on the characteristics of the patient cohort.

Fundus autofluorescence is proposed to be highly sensitive for the detection of neovascular conversion. ${ }^{13,17,40}$ Cachulo et al. ${ }^{13}$ report fundus autofluorescence and retinal leakage analysis as the most sensitive for conversion compared to other techniques (color fundus photography, fluorescein and indocyanine green angiography, and Stratus OCT). Fundus autofluorescence imaging showed a sensitivity of $93 \%$, specificity of $37 \%$, positive predictive value of $57 \%$, and negative predictive value of $93 \%$. The same authors used the IFAG scheme to stratify at-risk eyes with AMD and showed that having fundus autofluorescence abnormalities at baseline increased the incidence of conversion to neovascular AMD. However, no particular baseline fundus autofluorescence pattern correlated with conversion. ${ }^{13,17}$

In contrast, Batioglu et al. ${ }^{40}$ classified a cohort of early and intermediate AMD cases (featuring drusen and pigmentary changes) based on the predominant IFAG pattern and found a significantly higher frequency of choroidal neovascularization development in eyes with the patchy, linear, and reticular patterns (Fig. 3A-C). ${ }^{40}$ Hence, various patterns of fundus autofluorescence abnormalities 


\section{Fundus autofluorescence of intermediate age-related macular degeneration*}

Alterations that may be associated with a higher risk of conversion to choroidal neovascularization based on the work of Batioglu et al., ${ }^{40}$

Einbock et al. ${ }^{49}$ and the International Fundus Autofluorescence Classification Group ${ }^{27}$

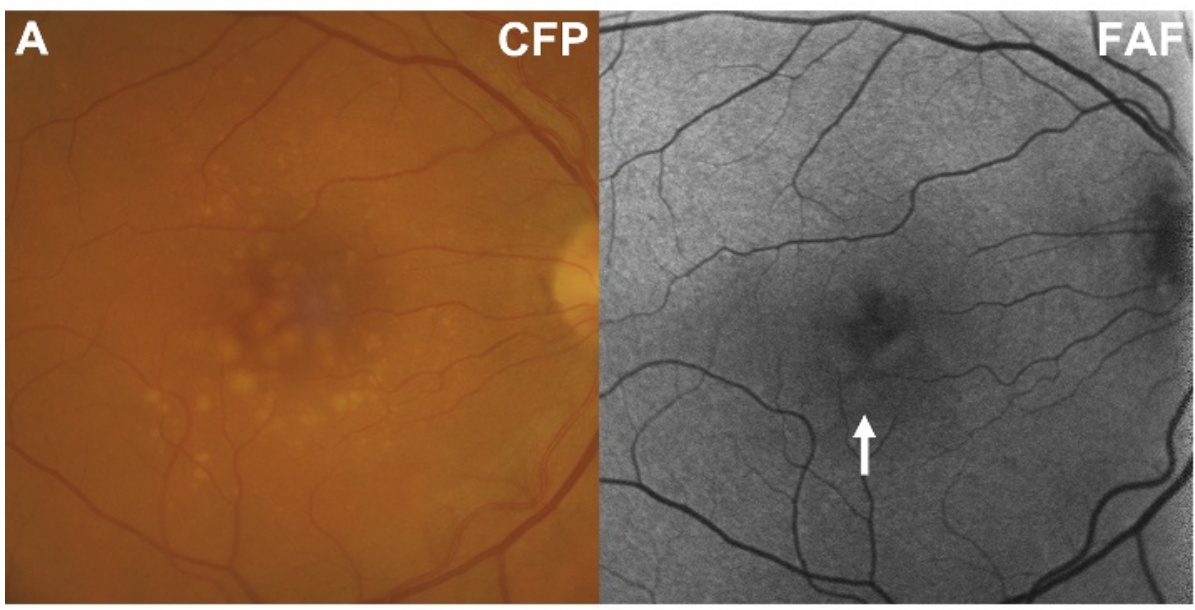

\section{Patchy pattern}

- Features at least one large area $(>200 \mu \mathrm{m}$ diameter) of markedly increased FAF

- Relatively ill-defined borders

- May correspond to large, soft drusen and hyper-pigmentary changes

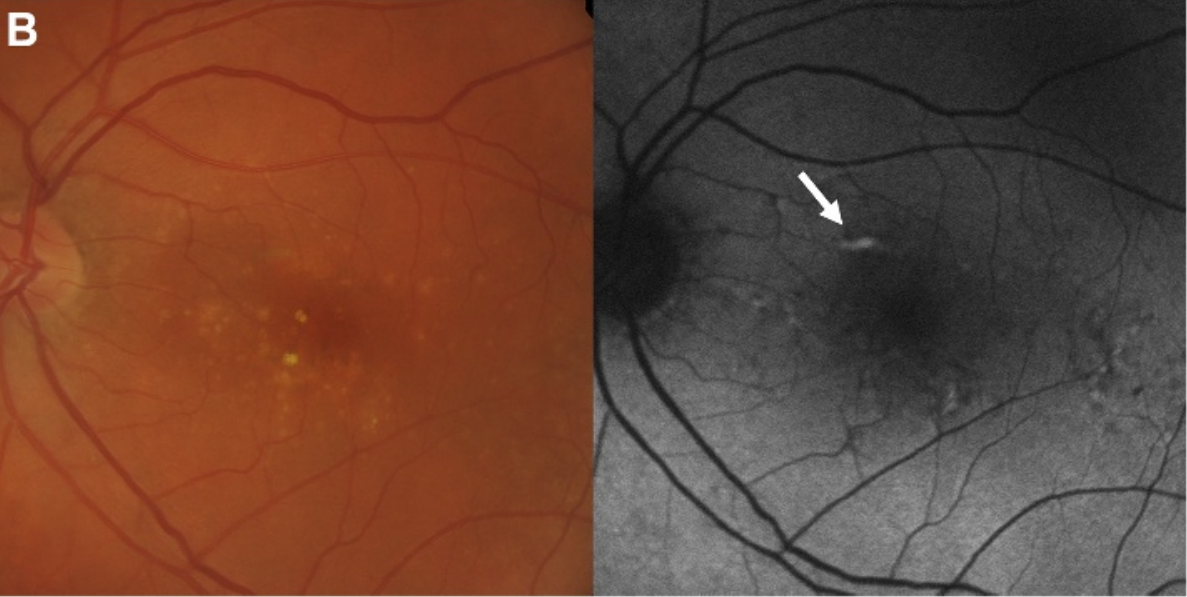

\section{Linear pattern}

- Features at least one linear area of markedly increased FAF

- Well-defined borders

- Often corresponds to hyper-pigmented lines on retinal photography

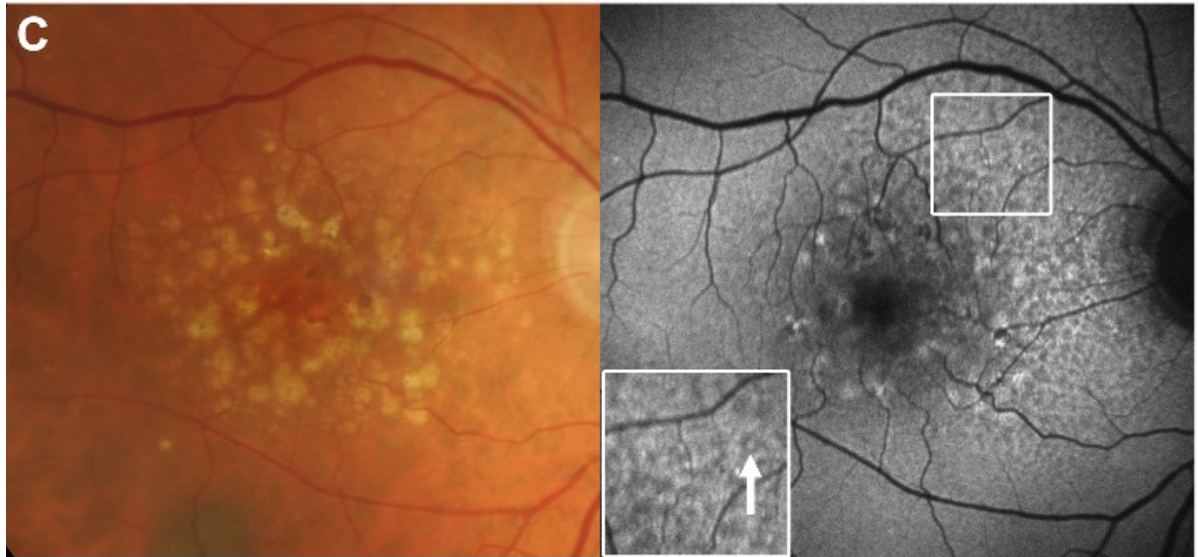

\section{Reticular pattern}

- Features multiple, small, round, regularly networked areas $(<200 \mu \mathrm{m}$ diameter) of decreased FAF

- Individual lesions may show a target appearance

* Based on the Beckman Initiative for Macular Research Classification scheme ${ }^{36}$

\section{FIGURE 3.}

Page one of a chair-side reference chart designed to aid in the differential diagnosis of AMD phenotypes using fundus autofluorescence (FAF) imaging. Conclusions regarding the prognostic utility of FAF imaging in intermediate AMD are still equivocal, and this should be considered as a limitation of this clinical chart. The phenotypes in intermediate AMD pictured may be associated with a higher risk of conversion to choroidal neovascularization. Further details are described under section 4. Stratification of early to intermediate AMD phenotypes using FAF. CFP, color fundus photograph; FAF, fundus autofluorescence. 


\section{Fundus autofluorescence of advanced age-related macular degeneration}
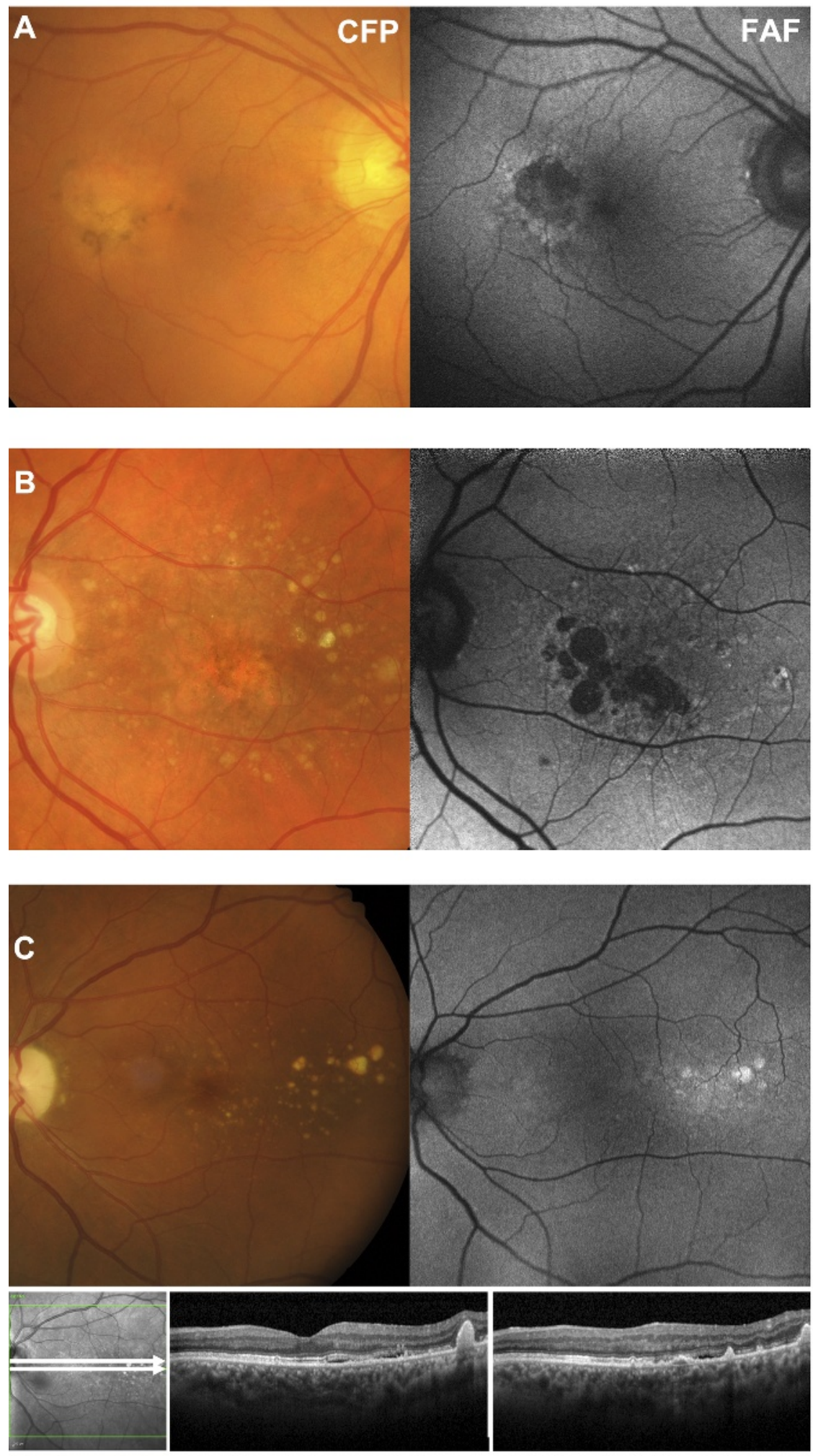

${ }^{*}$ Based on the Beckman Initiative for Macular Research Classification scheme ${ }^{36}$

** Evaluation for foveal involvement may be difficult without complementary imaging techniques ${ }^{15}$

\section{FIGURE 4.}

Second page of the chair-side reference showing different presentations of advanced AMD and the corresponding, typical appearance using fundus autofluorescence (FAF) imaging. Abbreviations as in Fig. 3.

\section{Uni-lobular geographic atrophy}

- Well-demarcated, single area of hypo-autofluorescence

- Foveal sparing** is characterised in this example by a symmetrical and gradual reduction in FAF approaching the fovea, as in normal eyes

\section{Multi-lobular geographic atrophy}

- The junctional zone in this case shows a diffuse trickling pattern, which has been associated with a relatively high rate of enlargement (compared to other patterns)

- Foveal sparing** in this example appeared as irregular hypo-autofluorescence of the

\section{Neovascular AMD}

- Increased FAF corresponds with subretinal fluid residual foveal island

- Irregular, mottled autofluorescence pattern 
can be observed in early and intermediate AMD, reflecting different stages and phenotypes that may hold prognostic value. ${ }^{37,40,48,49}$

\section{Fundus Autofluorescence Imaging of Late AMD}

Late AMD is defined as neovascular AMD and/or geographic atrophy. ${ }^{36}$ The RPE changes that define advanced states of AMD and disease progression are readily detectable using fundus autofluorescence imaging. ${ }^{12}$

\section{Geographic Atrophy}

The appearance of geographic atrophy was the subject of most articles regarding fundus autofluorescence imaging and AMD. Geographic atrophy appears as a well-demarcated region (unifocal or unilobular) or regions (multifocal or multilobular) of marked hypo-autofluorescence from an absence of the RPE (which contains the lipofuscin fluorophore). ${ }^{4,12,18,22,23,26,35,38-40,44}$ Geographic atrophy has a predilection for the central or parafoveal macula and may extend to involve areas of peripapillary atrophy. ${ }^{19}$ More typically, several patches in the parafovea may combine causing a "horse-shoe" or ring-type configuration. ${ }^{38}$ Consequently, areas of geographic atrophy may feature internal "spared" regions of retinal tissue ${ }^{35}$; new lobules may display a relatively lower intensity of hypo-autofluorescence, appearing more gray than black (Fig. 4A). ${ }^{44}$

Measurements of lesion size on fundus autofluorescence imaging show high inter-grader reproducibility ${ }^{15,26,35}$ due to the abrupt, high-contrast transition at lesion edges from hypo-autofluorescence to normal background fundus autofluorescence.$^{11,18,19,50}$ Fundus autofluorescence also better detects more discrete (especially small) areas of geographic atrophy in comparison to other modalities. ${ }^{35,44}$ Hence, fundus autofluorescence imaging is especially valuable for clinical cases of geographic atrophy undergoing surveillance ${ }^{18,22,35,38,50}$ and as an endpoint in clinical trials. ${ }^{15,51}$

\section{Foveal Involvement of Geographic Atrophy}

The degree of foveal involvement or sparing of atrophic areas may also be graded using fundus autofluorescence imaging. ${ }^{15,22}$ However, Sayegh et al..$^{15}$ reported relatively low inter-individual grader agreement (48/81 eyes; 59.3\%) using a four-point scale. Such evaluations are likely to be hampered by the symmetrical, hypo-autofluorescent pattern at the fovea that occurs in normal eyes, which may appear irregularly reduced in cases of parafoveal geographic atrophy with foveal sparing. ${ }^{18,38,52}$ Sensitivity and specificity values relating to the detection of foveal involvement using fundus autofluorescence imaging were 72 to $93 \%$ and 59 to $88 \%$, lower than color fundus photography (sensitivity: $86-97 \%$, specificity: 74-76\%). ${ }^{35}$ Consequently, screening for decreased fundus autofluorescence in the central fovea as a surrogate indicator for geographic atrophy may be difficult without complementary examination using another modality (such as infrared imaging; Fig. $4 \mathrm{~A}-\mathrm{C}) .{ }^{15}$

\section{Patterns of Fundus Autofluorescence in the Junctional Zone Surrounding Geographic Atrophy}

Hyper-autofluorescence surrounding geographic atrophy has also been observed in $32.5 \%^{12}$ to $85 \%^{11}$ of eyes, and varies in appearance from small, isolated spots to large irregularly shaped areas. ${ }^{23}$ These changes may be detected using either blue or green light excitation. ${ }^{18}$ The Fundus Autofluorescence in Age-related Macular Degeneration Group (FAM Study Group) ${ }^{22}$ characterized such abnormalities in the junctional zone of geographic atrophy areas, using four categories: (1) focal increased, (2) banded, (3) patchy, and (4) diffuse. Diffuse describes autofluorescence patterns that extend beyond the margins of the geographic atrophy and includes reticular, branching, fine granular, and fine granular with peripheral punctate spots subtypes. Similar junctional patterns have been described in other studies. ${ }^{9,11,18,22,23,26,52-54}$ They may exceed a $30 \times 30$ degree image frame and necessitate good image quality with even illumination and high signal-to-noise ratio for accurate identification. ${ }^{9,18,23}$ Speculated reasons for hyper-autofluorescence at the junctional zone surrounding geographic atrophy include RPE cell hypertrophy, shedding of RPE cells into the subretinal space, phagocytosis of melanin and cellular debris, or a combination of such processes. ${ }^{52}$ The findings often correlate with alterations in the outer retinal layers on $\mathrm{OCT}^{11,39,52}$ and imply a causal association between lipofuscin and geographic atrophy progression. ${ }^{53}$

Some junctional patterns have been associated with increased rate of progression. ${ }^{9,23,54} \mathrm{Schmitz}-$ Valckenberg et al. ${ }^{23}$ observed a positive correlation between the rate of geographic atrophy progression and the area of diffuse, hyper-fluorescence outside atrophic areas at baseline. Similarly, Holz et al. ${ }^{9}$ found progression of geographic atrophy was slowest in eyes with no surrounding abnormal fundus autofluorescence pattern and consecutively greater with different junctional patterns (focal followed by diffuse and banded). Those authors ${ }^{9}$ described an additional diffuse fundus autofluorescence pattern, termed "diffuse trickling" that included "speck-light, ramified, grainy, dusty and heterogeneous seeping" patterns, similar to that shown in Fig. 4B. In particular, atrophic areas in that group of nine eyes also appeared more gray than dark black. ${ }^{9}$ Fundus autofluorescence of atrophic areas in those eyes showed significantly higher rates of enlargement (an 8-fold difference, median enlargement rate of $3.02 \mathrm{~mm}^{2} / \mathrm{yr}$ ) compared to eyes without fundus autofluorescence abnormalities in the junctional zone $\left(0.38 \mathrm{~mm}^{2} / \mathrm{yr}\right)$; eyes featuring the other diffuse types had a median progression rate of $1.67 \mathrm{~mm}^{2} / \mathrm{yr}^{9}{ }^{9}$

Smith et al. ${ }^{4}$ described an evolutionary shift of lipofuscinassociated increased fundus autofluorescence overlying drusen to areas adjacent to drusen and geographic atrophy. The authors interpreted these patterns as suggesting that progression of disease may involve secondary dispersal and/or deposition of lipofuscin around geographic atrophy. ${ }^{4}$ However, the rate of progression in geographic atrophy (and associated visual morbidity) shows significant inter-individual variation, ${ }^{22,23}$ and there is mounting evidence that implicates non-focal hyper-autofluorescence (lipofuscinassociated toxicity) in the junctional zone of geographic atrophy as portending the development of new geographic atrophy. $9,22,23,52,54$

\section{Neovascular AMD}

Exudation in neovascular AMD results in the accumulation of various sub- and intraretinal materials including serous fluid, hemorrhage, lipid, and fibrovascular materials. ${ }^{55}$ Alterations in fundus autofluorescence corresponding with areas of choroidal neovascularization may be characterized by these inherent features 
as follows. Subretinal fluid corresponds with increased fundus autofluorescence in approximately $56.5 \%$ of cases (Fig. 4C). ${ }^{12,29}$ Discifom scarring consistently demonstrates uneven hypo-autofluorescence of the lesion, surrounded by marked hyper-autofluorescence. ${ }^{12,29}$ Hemorrhages, ${ }^{29,56,57}$ exudate, ${ }^{57}$ and fibrovascular membranes ${ }^{30}$ are also likely to cause hypo-autofluorescence patterns. RPE tears show decreased fundus autofluorescence, indicating denuded RPE with surrounding hyper-autofluorescent margins. The hypo-autofluorescent areas may evolve after 3 months to hazy or speckled hyperautofluorescence in approximately $20 \%$ of cases. ${ }^{58}$ Unaffected regions outside neovascular lesions are likely to display a typical macular pattern of homogeneous fundus autofluorescence. ${ }^{59}$ In untreated, early-stage subfoveal neovascular AMD, fundus autofluorescence is often continuous at the macula (found in 54/79 eyes studied $\left.{ }^{10}\right)$. A novel finding of subretinal material associated with neovascular AMD, termed subretinal hyperreflective exudation, has also been characterized as iso- or hypo-autofluorescent and appears distinct from other subretinal materials such as hemorrhage. ${ }^{55}$
Using angiographic characterizations, classic choroidal neovascularization often presents (in 79-90\% of cases) with corresponding hypo-autofluorescence, which may be surrounded by a thin halo of hyper-AF in 34 to $50 \%$ of cases. ${ }^{56,57,59}$ The halo or ring seen using fundus autofluorescence imaging in classic choroidal neovascularization likely relates to the hyperplastic proliferation of RPE cells around the lesion ${ }^{56,57,59}$ or the presence of macrophages. ${ }^{57}$ In contrast, fundus autofluorescence images of occult choroidal neovascularization show irregular (both increased and decreased) fundus autofluorescence signal intensity, with multiple foci of hypo-autofluorescence in at least $50 \%$ of cases ${ }^{57,59}$ and no identifiable junctional zone. ${ }^{56}$ These foci have been described as relating to small, heterogeneous areas of RPE damage or loss. ${ }^{59} \mathrm{~A}$ mixed presentation (with diffuse hypo-autofluorescent lesions surrounded by irregular, heterogeneous regions of autofluorescence) may also occur. ${ }^{56,57}$ Type 3 (retinal angiomatous proliferation) neovascularization in AMD shows discrete, uniform hypo-autofluorescence coincident with the lesion site, associated with masking by small retinal hemorrhages. ${ }^{48}$
UWFI
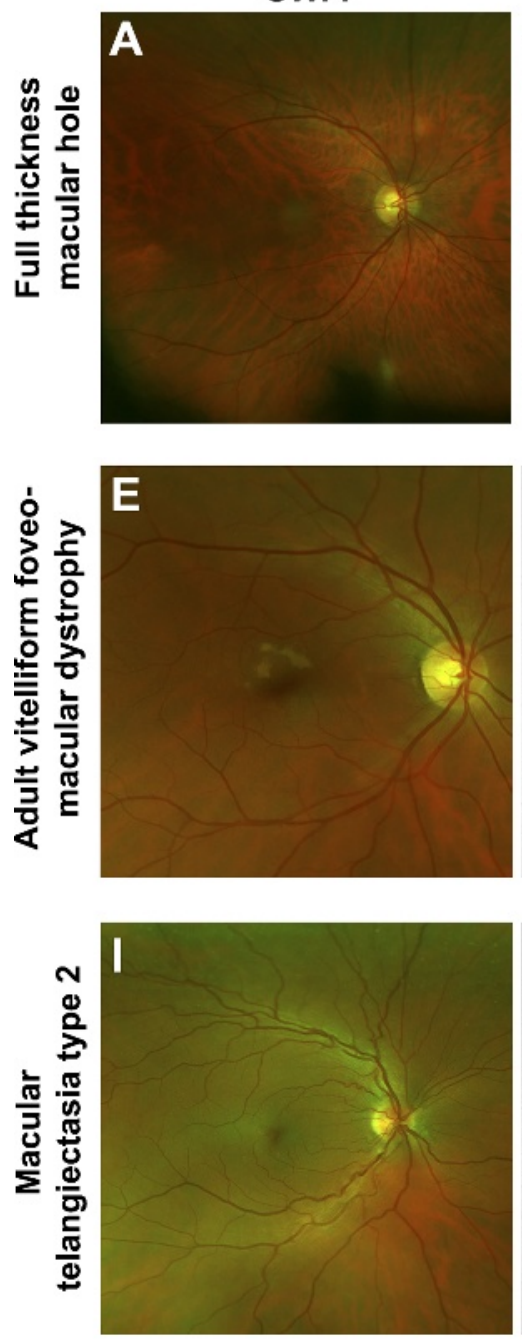

FAF
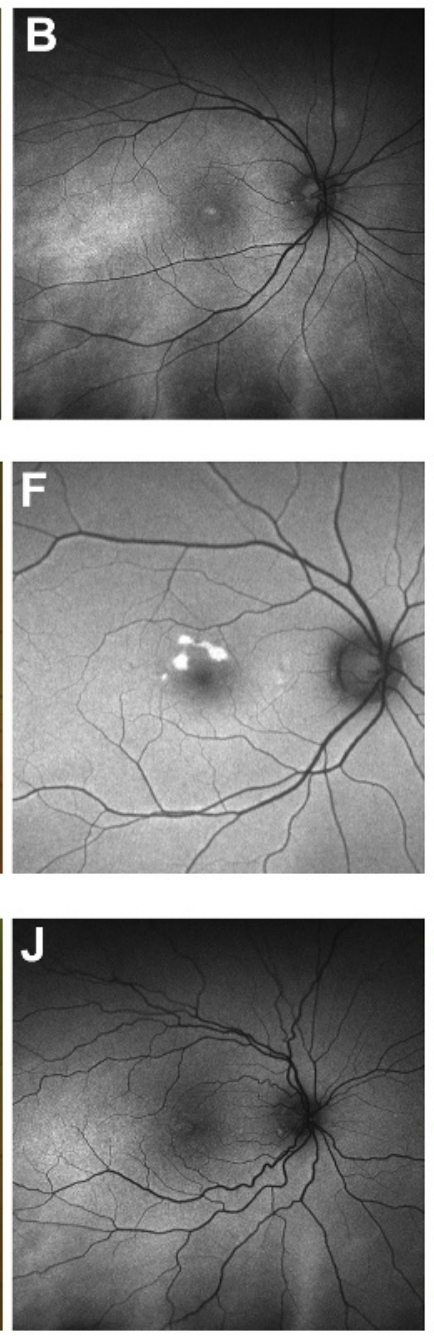

IR
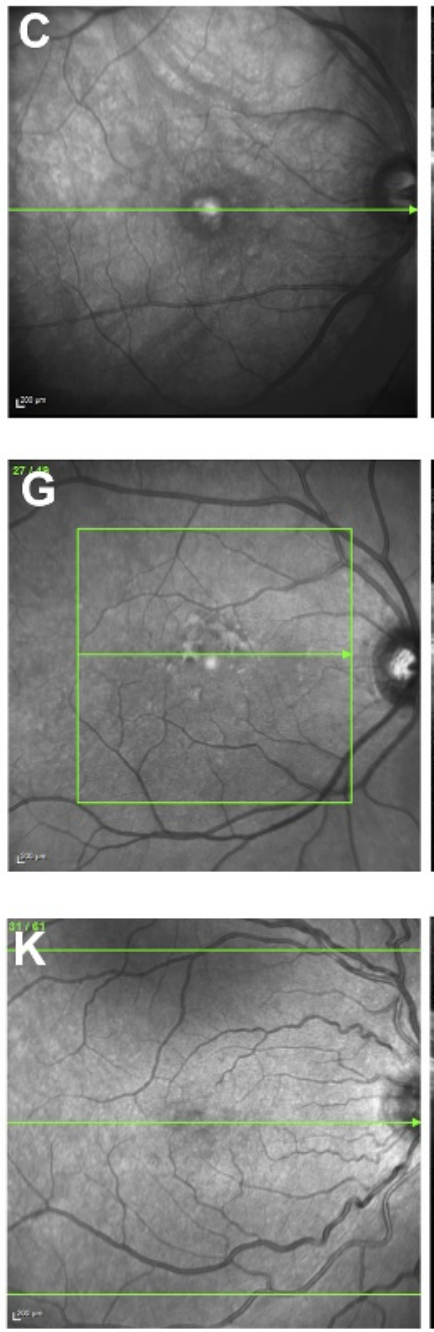
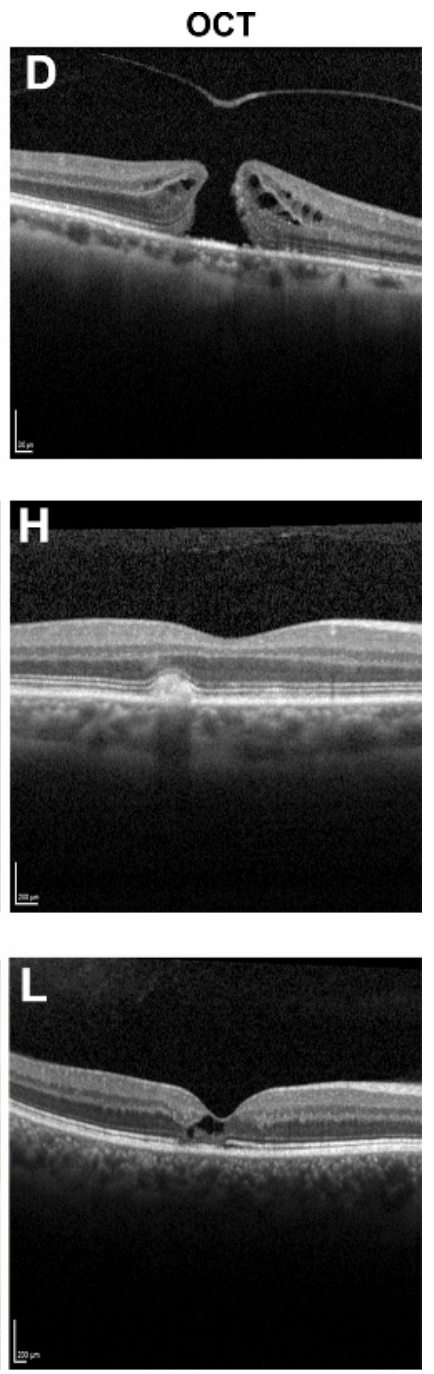

\section{FIGURE 5.}

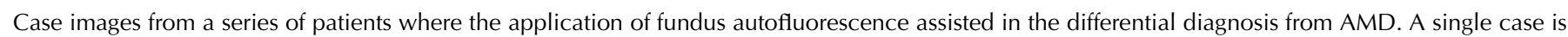

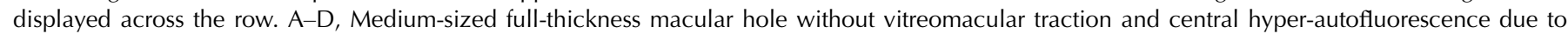

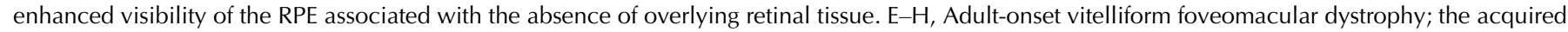

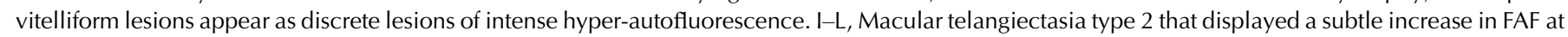
the temporal fovea. Abbreviations as in Fig. 1. IR, infrared reflectance image; OCT, optical coherence tomography. 
Eyes with neovascular AMD (regardless of subtype - classic or occult) can also present with normal or near normal fundus autofluorescence imaging results. ${ }^{4,59}$ Preservation of normal fundus autofluorescence in neovascular AMD indicates that the RPE and photoreceptor complex remain intact and may appear so for several months after the onset of choroidal neovascularization. Alternatively, fundus autofluorescence has also been described as a noninvasive method of evaluating AMD-related RPE loss in eyes with choroidal neovascularization, which often presents at the macular center in initial stages and expands outward. ${ }^{51}$ This is in contrast with geographic atrophy, which usually develops in an extrafoveal location. Thus, fundus autofluorescence imaging may be useful for monitoring and for determining which eyes are likely to benefit from therapeutic intervention. ${ }^{10,57}$ In other cases, the relatively large spans of hypo-autofluorescence seen in neovascular AMD are related to a blockage of fundus autofluorescence (rather than RPE damage) by subretinal fluid or the fibrovascular membrane itself (blood or choroidal neovascularization). ${ }^{12,56,59}$ Furthermore, the incidence of normal fundus autofluorescence patterns (and rare observations of hyper-autofluorescence preceding choroidal neovascularization) suggests that neovascular cases may not involve abnormal accumulations of lipofuscin. ${ }^{4,40,59}$

\section{Differential Diagnosis of AMD Using Fundus Autofluorescence}

Fundus autofluorescence imaging has been described in the distinction of AMD from other macular conditions and dystrophies (Fig. 5) including lesions associated with more widespread fundus changes (Fig. 6).

\section{Macular Dystrophies}

Late-onset macular dystrophies such as central areolar choroidal dystrophy and Stargardt's disease can be misdiagnosed as AMD due to their overlapping presentation and low prevalence. ${ }^{60} \mathrm{In}$ addition, incomplete disease penetrance in central areolar choroidal dystrophy means the autosomal dominant mode of inheritance may not be revealed in the patient's family history. ${ }^{60,61}$

More specifically, the characteristic yellow fundus flecks of late onset Stargardt's disease/fundus flavimaculatus display intense hyper-autofluorescence (Fig. 6A-C), which is greater than drusen in AMD, more irregular in shape, and diffusely spread. ${ }^{62}$ Thirtyseven percent of late-onset Stargardt's disease cases also show mottled hypo-autofluorescence at the macula. ${ }^{62}$ AMD-related fundus autofluorescence patterns described as "speckled" and "fine granular with punctuated spots" closely resemble the fundus autofluorescence pattern of Stargardt's disease and may be misdiagnosed. ${ }^{62}$

Central areolar choroidal dystrophy lesions, which can mimic AMD-related geographic atrophy, generally demonstrate a slower rate of enlargement $\left(0.61 \mathrm{~mm}^{2}\right.$ over 18 months compared to $1.52 \mathrm{~mm}^{2}$ per year) on fundus autofluorescence imaging. Similarly, drusen-like changes at the edge of central areolar choroidal dystrophy lesions show intense hyper-autofluorescence, which is atypical of drusen in AMD. ${ }^{61}$ Speckled patterns of hyper- and hypoautofluorescence described in both conditions can be distinguished using prevalence $(85 \%$ of central areolar choroidal dystrophy cases versus $5.6 \%$ of early AMD cases) and appearance: relatively well demarcated, regular in shape (round or oval), and centrally located in central areolar choroidal dystrophy compared to AMD ${ }^{60}$ A reticular hypo-autofluorescence pattern or reticular pseudodrusen is associated with up to $93 \%$ of late AMD cases with geographic atrophy ${ }^{44}$ and is not observed in central areolar choroidal dystrophy. ${ }^{60}$

\section{Other Macular Conditions}

In a macular hole (Fig. 5A-D), increased fundus autofluorescence centrally is a common finding that has been attributed to enhanced visibility of the RPE from lack of overlying neurosensory retina. ${ }^{56}$ Central serous chorioretinopathy (Fig. 6D-F) in fundus autofluorescence images is most easily distinguished from AMD by atrophic gravitational tracks. ${ }^{47}$ Multimodal imaging of malattia leventinese (autosomal dominant drusen; Fig. 6G-I) reveals similar fundus autofluorescence characteristics of drusen in AMD. ${ }^{63}$ Consequently, the differential diagnosis of malattia leventinese presently relies on the earlier age at presentation (typically before the age of 50 years) and characteristic radial distribution of drusen temporal to the macula. Acquired vitelliform lesions (Fig. $5 \mathrm{E}-\mathrm{H}$ ) exhibit intense hyper-autofluorescence, which is atypical of AMD. ${ }^{55}$ In contrast, macular telangiectasia type 2 (Fig. 5I-L) displays a more subtle increase in fundus autofluorescence at the temporal fovea, related to a "depletion" of macular pigment. ${ }^{64}$

Thus, certain macular disorders (macular dystrophies, macular hole, central serous chorioretinopathy, and acquired vitelliform lesions) may be more amenable to fundus autofluorescence-assisted differential diagnosis due to their consistent autofluorescence characteristics. However, others such as malattia leventinese and retinitis pigmentosa (Fig. 6J-L) may require more careful consideration of the overall clinical picture. For more discourse relating to the differential diagnosis of each entity from AMD, the reader is recommended to reviews by Dosunmu and Bakri, ${ }^{65}$ and Saksens et al. ${ }^{66}$

\section{CONCLUSIONS}

The wide variation in fundus autofluorescence patterns in AMD is consistent with the heterogeneity (clinical variability) of the disease itself. ${ }^{12}$ Fundus autofluorescence imaging has the capacity to reveal RPE alterations in areas that appear normal on funduscopy in early AMD and may have prognostic implications. It also represents a reliable method for distinguishing reticular pseudodrusen and can assist in the differential diagnosis of AMD from other disorders. ${ }^{43}$

Furthermore, fundus autofluorescence offers significant utility in the detection, evaluation, and monitoring of atrophic AMD. This is particularly useful given that grading areas of geographic atrophy using color fundus photography may be laborious and difficult due to inter-individual variations in fundus pigmentation, and the presence of small satellite lesions. ${ }^{35}$ Geographic atrophy features highly variable, inter-individual rates of progression. Risk for progression is presently evaluated by considering the baseline size of the atrophic area and characteristics of the fellow eye (large drusen and pigmentary changes). Consequently, if the prognostic value of fundus autofluorescence imaging holds true, the technique may hold a stronger ${ }^{54}$ and invaluable role in assessing increased risk of 

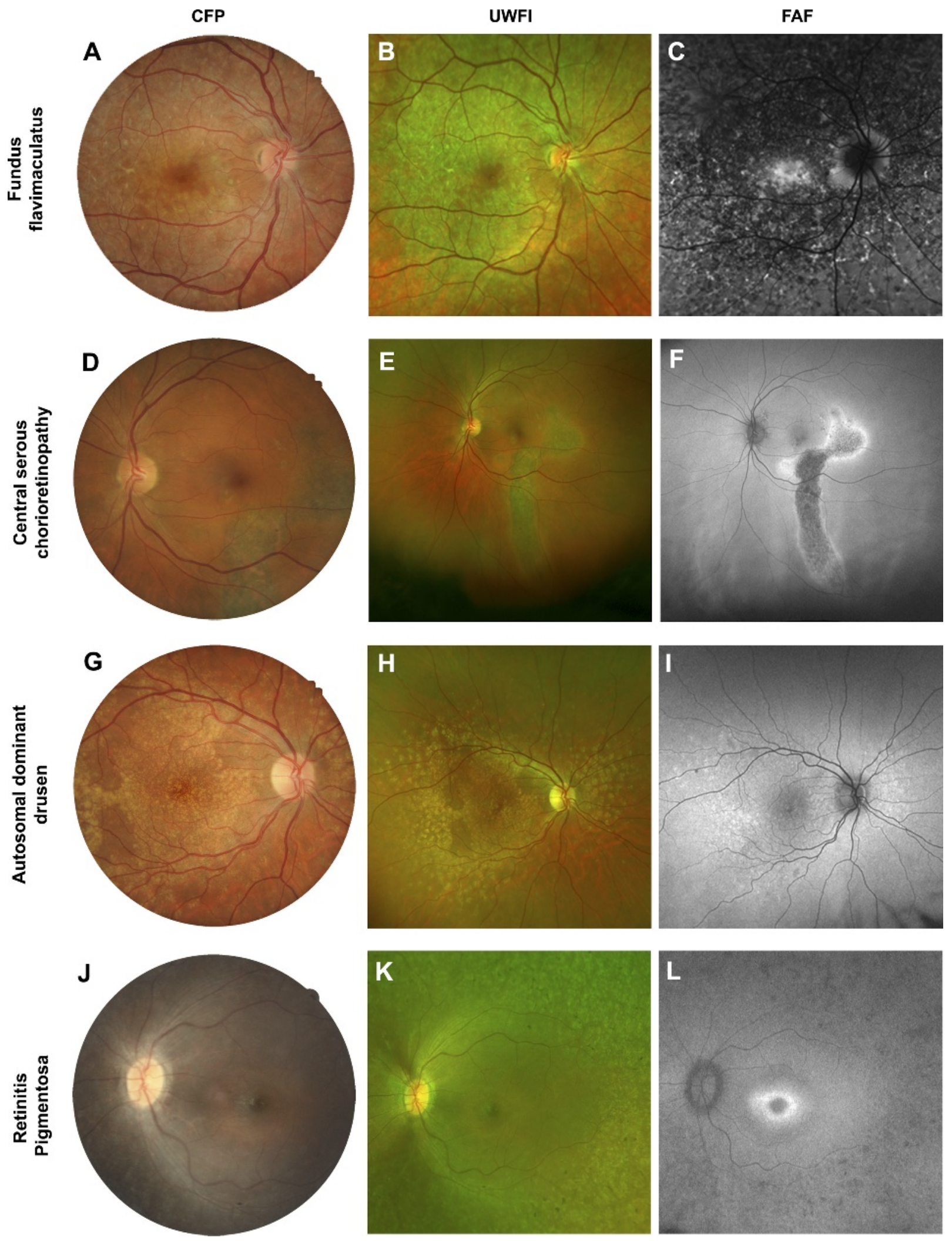

\section{FIGURE 6.}

Case series from patients that featured more extensive fundus changes involving the macula. A single case is displayed across the row. A-C, Fundus flavimaculatus: the characteristic flecks displayed prominent hyper-autofluorescence. D-F, Central serous chorioretinopathy distinguished by the typical appearance of a gravitational atrophic tract. G-I, Autosomal dominant drusen which appeared as discrete, radially distributed areas of hyperautofluorescence. J-L, Retinitis pigmentosa characterized by patchy hypofluorescence in the periphery and a hyper-autofluorescent ring at the fovea. Abbreviations as in Fig. 1. 
incipient geographic atrophy in AMD. ${ }^{9}$ In neovascular AMD, fundus autofluorescence imaging reveals distinct patterns of classic choroidal neovascularization noninvasively and may be especially useful for determining which eyes are likely to benefit from therapeutic intervention. ${ }^{25,58}$

In conclusion, research efforts dedicated to the evaluation of fundus autofluorescence imaging in AMD are vast. Fundus autofluorescence imaging represents a rapid, effective, noninvasive imaging method that has significant applicability. Future efforts to evaluate how well these tools are presently incorporated into clinical practice are currently underway.

\section{ACKNOWLEDGMENTS}

This work was supported, in part, by grants and awards from the University of New South Wales (Early Career Research Grant 2015 \#P535430), an Australian Postgraduate Award, and a National Health and Medical Research Council (NHMRC) grant (\#1033224). Guide Dogs NSWIACT is a partner in the NHMRC grant and also provided a supplementary $P h D$ scholarship for $A L$ and support for $L N-S$.

The authors report no conflicts of interest and have no proprietary interest in any of the instruments mentioned in this article.

Received January 7, 2016; accepted June 15, 2016.

\section{REFERENCES}

1. Wong WL, Su X, Li X, et al. Global prevalence of age-related macular degeneration and disease burden projection for 2020 and 2040: a systematic review and meta-analysis. Lancet Glob Health 2014;2: e106-16.

2. Nivison-Smith L, Milston R, Madigan M, et al. Age-related macular degeneration: linking clinical presentation to pathology. Optom Vis Sci 2014;91:832-48.

3. von Ruckmann A, Fitzke FW, Bird AC. Distribution of fundus autofluorescence with a scanning laser ophthalmoscope. $\mathrm{Br} \mathrm{J}$ Ophthalmol 1995;79:407-12.

4. Smith RT, Chan JK, Busuoic M, et al. Autofluorescence characteristics of early, atrophic, and high-risk fellow eyes in age-related macular degeneration. Invest Ophthalmol Vis Sci 2006;47: 5495-504.

5. American Optometric Association Consensus Panel on Care of the Patient with Age-Related Macular Degeneration. Optometric Clinical Practice Guideline. Care of the Patient with Age-Related Macular Degeneration; 2004. Available at: http://www.aoa.org/documents/ optometrists/CPG-6.pdf. Accessed: January 4, 2016.

6. The Royal College of Ophthalmologists. Age-Related Macular Degeneration: Guidelines for Management; September 2013. Available at: https:/www.rcophth.ac.uk/wp-content/uploads/2014/12/2013SCI-318-RCOphth-AMD-Guidelines-Sept-2013-FINAL-2.pdf. Accessed: January 4, 2016.

7. American Academy of Ophthalmology Retina/Vitreous Panel 2013-2014. Preferred Practice Pattern ${ }^{\circledR}$ Guidelines. Age-Related Macular Degeneration. San Francisco, CA, 2015. Available at: http:// www.aao.org/Assets/db935a77-1997-4d60-b850-71b7602f46e2/ 635582143853270000/age-related-macular-degeneration-ppppdf. Accessed: January 4, 2016.

8. Canadian Journal of Optometry/Revue Canadienne d'Optométrie (CJO RCO). Guidelines for the Collaborative Management of Persons with Age-Related Macular Degeneration by Health- and Eye-Care Professionals; 2015. Available at: https:/opto.ca/sites/ default/files/resources/documents/cjo_journal_online_ehco_ guidelines_eng_v2.pdf. Accessed: January 4, 2016.

9. Holz FG, Bindewald-Wittich A, Fleckenstein M, et al. Progression of geographic atrophy and impact of fundus autofluorescence patterns in age-related macular degeneration. Am J Ophthalmol 2007;143: 463-72.

10. Vaclavik V, Vujosevic S, Dandekar SS, et al. Autofluorescence imaging in age-related macular degeneration complicated by choroidal neovascularization: a prospective study. Ophthalmology 2008;115: 342-6.

11. Wolf-Schnurrbusch UE, Enzmann V, Brinkmann CK, et al. Morphologic changes in patients with geographic atrophy assessed with a novel spectral OCT-SLO combination. Invest Ophthalmol Vis Sci 2008;49:3095-9.

12. Kellner U, Kellner S, Weinitz S. Fundus autofluorescence (488 NM) and near-infrared autofluorescence $(787 \mathrm{NM})$ visualize different retinal pigment epithelium alterations in patients with age-related macular degeneration. Retina 2010;30:6-15.

13. Cachulo L, Silva R, Fonseca P, et al. Early markers of choroidal neovascularization in the fellow eye of patients with unilateral exudative age-related macular degeneration. Ophthalmologica 2011;225:144-9.

14. Querques G, Querques L, Martinelli D, et al. Pathologic insights from integrated imaging of reticular pseudodrusen in age-related macular degeneration. Retina 2011;31:518-26.

15. Sayegh RG, Simader C, Scheschy U, et al. A systematic comparison of spectral-domain optical coherence tomography and fundus autofluorescence in patients with geographic atrophy. Ophthalmology 2011;118:1844-51.

16. Schmitz-Valckenberg S, Alten F, Steinberg JS, et al. Reticular drusen associated with geographic atrophy in age-related macular degeneration. Invest Ophthalmol Vis Sci 2011;52:5009-15.

17. Silva R, Cachulo ML, Fonseca P, et al. Age-related macular degeneration and risk factors for the development of choroidal neovascularisation in the fellow eye: a 3-year follow-up study. Ophthalmologica 2011;226:110-8.

18. Wolf-Schnurrbusch UE, Wittwer VV, Ghanem R, et al. Blue-light versus green-light autofluorescence: lesion size of areas of geographic atrophy. Invest Ophthalmol Vis Sci 2011;52:9497-502.

19. Mauschitz MM, Fonseca S, Chang P, et al. Topography of geographic atrophy in age-related macular degeneration. Invest Ophthalmol Vis Sci 2012;53:4932-9.

20. Tan CS, Heussen F, Sadda SR. Peripheral autofluorescence and clinical findings in neovascular and non-neovascular age-related macular degeneration. Ophthalmology 2013;120:1271-7.

21. Toy BC, Krishnadev N, Indaram M, et al. Drusen regression is associated with local changes in fundus autofluorescence in intermediate age-related macular degeneration. Am J Ophthalmol 2013; 156:532-42.e1.

22. Bindewald A, Schmitz-Valckenberg S, Jorzik JJ, et al. Classification of abnormal fundus autofluorescence patterns in the junctional zone of geographic atrophy in patients with age related macular degeneration. Br J Ophthalmol 2005;89:874-8.

23. Schmitz-Valckenberg S, Bindewald-Wittich A, Dolar-Szczasny J, et al. Correlation between the area of increased autofluorescence surrounding geographic atrophy and disease progression in patients with AMD. Invest Ophthalmol Vis Sci 2006;47:2648-54.

24. Hogg RE, Silva R, Staurenghi G, et al. Clinical characteristics of reticular pseudodrusen in the fellow eye of patients with unilateral neovascular age-related macular degeneration. Ophthalmology 2014; 121:1748-55. 
25. Fujimura S, Ueta T, Takahashi $\mathrm{H}$, et al. Characteristics of fundus autofluorescence and drusen in the fellow eyes of Japanese patients with exudative age-related macular degeneration. Graefes Arch Clin Exp Ophthalmol 2013;251:1-9.

26. Bearelly S, Khanifar AA, Lederer DE, et al. Use of fundus autofluorescence images to predict geographic atrophy progression. Retina 2011;31:81-6.

27. Bindewald A, Bird AC, Dandekar SS, et al. Classification of fundus autofluorescence patterns in early age-related macular disease. Invest Ophthalmol Vis Sci 2005;46:3309-14.

28. Landa G, Rosen RB, Pilavas J, et al. Drusen characteristics revealed by spectral-domain optical coherence tomography and their corresponding fundus autofluorescence appearance in dry age-related macular degeneration. Ophthalmic Res 2012;47:81-6.

29. Vujosevic S, Vaclavik V, Bird AC, et al. Combined grading for choroidal neovascularisation: colour, fluorescein angiography and autofluorescence images. Graefes Arch Clin Exp Ophthalmol 2007; 245:1453-60.

30. Yamamoto M, Kohno T, Shiraki K. Comparison of fundus autofluorescence of age-related macular degeneration between a fundus camera and a confocal scanning laser ophthalmoscope. Osaka City Med J 2009;55:19-27.

31. Spaide RF. Fundus autofluorescence and age-related macular degeneration. Ophthalmology 2003;110:392-9.

32. Reznicek L, Wasfy T, Stumpf C, et al. Peripheral fundus autofluorescence is increased in age-related macular degeneration. Invest Ophthalmol Vis Sci 2012;53:2193-8.

33. Witmer MT, Kozbial A, Daniel S, et al. Peripheral autofluorescence findings in age-related macular degeneration. Acta Ophthalmol 2012; 90:e428-33.

34. Pilotto E, Sportiello P, Alemany-Rubio E, et al. Confocal scanning laser ophthalmoscope in the retromode imaging modality in exudative age-related macular degeneration. Graefes Arch Clin Exp Ophthalmol 2013;251:27-34.

35. Khanifar AA, Lederer DE, Ghodasra JH, et al. Comparison of color fundus photographs and fundus autofluorescence images in measuring geographic atrophy area. Retina 2012;32:1884-91.

36. Ferris FL, 3rd, Wilkinson CP, Bird A, et al, Beckman Initiative for Macular Research Classification Committee. Clinical classification of age-related macular degeneration. Ophthalmology 2013;120:844-51.

37. Xuan Y, Zhao PQ, Peng Q. Fundus autofluorescence patterns of drusen in age-related macular degeneration. Zhonghua Yan $\mathrm{Ke} \mathrm{Za}$ Zhi 2010;46:708-13.

38. Schmitz-Valckenberg S, Fleckenstein M, Helb HM, et al. In-vivo imaging of foveal sparing in geographic atrophy secondary to agerelated macular degeneration. Invest Ophthalmol Vis Sci 2009;50: 3915-21.

39. Forte R, Querques G, Querques L, et al. Multimodal imaging of dry age-related macular degeneration. Acta Ophthalmol 2012;90:e281-7.

40. Batioglu F, Demirel S, Ozmert E, et al. Autofluorescence patterns as a predictive factor for neovascularization. Optom Vis Sci 2014; 91:950-5.

41. Ueda-Arakawa N, Ooto S, Tsujikawa A, et al. Sensitivity and specificity of detecting reticular pseudodrusen in multimodal imaging in Japanese patients. Retina 2013;33:490-7.

42. Smith RT, Sohrab MA, Busuioc M, et al. Reticular macular disease. Am J Ophthalmol 2009;148:733-43.e2.

43. Sarks J, Arnold J, Ho IV, et al. Evolution of reticular pseudodrusen. Br J Ophthalmol 2011;95:979-85.
44. Xu L, Blonska AM, Pumariega NM, et al. Reticular macular disease is associated with multilobular geographic atrophy in age-related macular degeneration. Retina 2013;33:1850-62.

45. Ueda-Arakawa N, Ooto S, Nakata I, et al. Prevalence and genomic association of reticular pseudodrusen in age-related macular degeneration. Am J Ophthalmol 2013;155:260-9.e2.

46. Lee MY, Ham DI. Subretinal drusenoid deposits with increased autofluorescence in eyes with reticular pseudodrusen. Retina 2014;34: 69-76.

47. Fung AT, Yannuzzi LA, Freund KB. Type 1 (sub-retinal pigment epithelial) neovascularization in central serous chorioretinopathy masquerading as neovascular age-related macular degeneration. Retina 2012;32:1829-37.

48. Querques G, Querques L, Forte R, et al. Precursors of type 3 neovascularization: a multimodal imaging analysis. Retina 2013;33: 1241-8.

49. Einbock W, Moessner A, Schnurrbusch UE, et al. Changes in fundus autofluorescence in patients with age-related maculopathy. Correlation to visual function: a prospective study. Graefes Arch Clin Exp Ophthalmol 2005;243:300-5.

50. Schmitz-Valckenberg S, Fleckenstein M, Gobel AP, et al. Optical coherence tomography and autofluorescence findings in areas with geographic atrophy due to age-related macular degeneration. Invest Ophthalmol Vis Sci 2011;52:1-6.

51. Kumar N, Mrejen S, Fung AT, et al. Retinal pigment epithelial cell loss assessed by fundus autofluorescence imaging in neovascular agerelated macular degeneration. Ophthalmology 2013;120:334-41.

52. Brar M, Kozak I, Cheng L, et al. Correlation between spectraldomain optical coherence tomography and fundus autofluorescence at the margins of geographic atrophy. Am J Ophthalmol 2009; 148:439-44.

53. Biarnes $\mathrm{M}$, Mones J, Trindade $\mathrm{F}$, et al. Intra and interobserver agreement in the classification of fundus autofluorescence patterns in geographic atrophy secondary to age-related macular degeneration. Graefes Arch Clin Exp Ophthalmol 2012;250:485-90.

54. Batioglu F, Gedik Oguz Y, Demirel S, et al. Geographic atrophy progression in eyes with age-related macular degeneration: role of fundus autofluorescence patterns, fellow eye and baseline atrophy area. Ophthalmic Res 2014;52:53-9.

55. Shah VP, Shah SA, Mrejen S, et al. Subretinal hyperreflective exudation associated with neovascular age-related macular degeneration. Retina 2014;34:1281-8.

56. Framme C, Bunse A, Sofroni R, et al. Fundus autofluorescence before and after photodynamic therapy for choroidal neovascularization secondary to age-related macular degeneration. Ophthalmic Surg Lasers Imaging 2006;37:406-14.

57. Peng Q, Dong Y, Zhao PQ. Fundus autofluorescence in exudative age-related macular degeneration. Genet Mol Res 2013;12:6140-8.

58. Caramoy A, Fauser S, Kirchhof B. Fundus autofluorescence and spectral-domain optical coherence tomography findings suggesting tissue remodelling in retinal pigment epithelium tear. $\mathrm{Br} \mathrm{J}$ Ophthalmol 2012;96:1211-6.

59. McBain VA, Townend J, Lois N. Fundus autofluorescence in exudative age-related macular degeneration. Br J Ophthalmol 2007;91: 491-6.

60. Smailhodzic D, Fleckenstein M, Theelen T, et al. Central areolar choroidal dystrophy (CACD) and age-related macular degeneration (AMD): differentiating characteristics in multimodal imaging. Invest Ophthalmol Vis Sci 2011;52:8908-18.

61. Boon CJ, Klevering BJ, Cremers FP, et al. Central areolar choroidal dystrophy. Ophthalmology 2009;116:771-82, 782.e1. 
62. Westeneng-van Haaften SC, Boon CJ, Cremers FP, et al. Clinical and genetic characteristics of late-onset Stargardt's disease. Ophthalmology 2012;119:1199-210.

63. Querques G, Guigui B, Leveziel N, et al. Multimodal morphological and functional characterization of Malattia Leventinese. Graefes Arch Clin Exp Ophthalmol 2013;251:705-14.

64. Sallo FB, Leung I, Clemons TE, et al. Multimodal imaging in type 2 idiopathic macular telangiectasia. Retina 2015;35:742-9.

65. Dosunmu EO, Bakri SJ. Mimickers of age-related macular degeneration. Semin Ophthalmol 2011;26:209-15.
66. Saksens NT, Fleckenstein M, Schmitz-Valckenberg S, et al. Macular dystrophies mimicking age-related macular degeneration. Prog Retin Eye Res 2014;39:23-57.

Michael Kalloniatis

Centre for Eye Health University of New South Wales Australia Sydney, NSW 2052 Australia e-mail:m.kalloniatis@unsw.edu.au 